\title{
A Virtual Subnetwork Approach to Earthquake Early Warning
}

\author{
by Yih-Min $\mathrm{Wu}$ and Ta-liang Teng
}

\begin{abstract}
Progress has been made toward the goal of earthquake early warning in Taiwan. By applying the concept of a virtual subnetwork (VSN) to the Taiwan Central Weather Bureau seismic network, the earthquake rapid-reporting time has been reduced to about $30 \mathrm{sec}$ or less by a new system called VSN. This represents a significant step toward realistic earthquake early-warning capability. The VSN system described here was put into operation from December 2000 to June 2001. A total of 54 earthquakes (100\% correct detection) were detected and processed successfully during this period. Comprehensive earthquake reports are issued mostly in less than $30 \mathrm{sec}$, with an average of about $22 \mathrm{sec}$ after the origin time. The 22 -sec reporting time will offer more than $20 \mathrm{sec}$ of early-warning time to cities at distances greater than $145 \mathrm{~km}$ from the source, for which the shear-wave strong-shaking arrival time is about $44 \mathrm{sec}$. This $20 \mathrm{sec}$ is an adequate amount of time to carry out numerous preprogrammed emergency-response measures prior to the arrival of strong shaking.
\end{abstract}

\section{Introduction}

Located on the western circum-Pacific seismic belt with a measured plate-boundary-convergence rate of about $8 \mathrm{~cm} /$ yr, Taiwan has been repeatedly hit by damaging earthquakes. Some of the damaging events have inflicted severe casualties and property losses. For example, on 17 March 1906 a damaging earthquake $(M 7.1)$ occurred in Chiayi $(1,258$ casualties, 2,385 injuries, 6,767 houses destroyed, and 15,218 houses damaged; Hsu, 2001). In 1935 a disastrous earthquake ( $M 7.1)$ occurred in the Hsinchu-Taichung area (3,276 casualties, 12,053 injuries, 17,907 houses destroyed, and 37,781 houses damaged; Hsu, 2001). The 1999 Chi-Chi earthquake $\left(M_{\mathrm{W}} 7.6\right)$ occurred in Nantou County $(2,456$ casualties, 11,306 injuries, with $\$ 4$ billion in property damage). This seismic loss potential will continue to exist and in fact will increase as the population grows. It is therefore crucial for Taiwan (and for many other parts of the world subject to seismic hazard) to seek means through scientific research to offset future earthquake losses.

Because of the extreme complexity involved in earthquake processes, reliable earthquake prediction is not currently possible (Kanamori et al., 1997). Present technological advances in seismic instrumentation, digital communication, and processing permit the implementation of a real-time earthquake-monitoring system. This system should be able to perform the functions of both rapid reporting and early warning (United States National Research Council, 1991). In Taiwan, research programs on earthquake rapidreporting systems (RRS) and early-warning systems (EWS) are being actively pursued. Some recent findings show that the RRS and EWS are becoming promising tools that can be made reliable enough to provide critical information useful in mitigating seismic hazards (Teng et al., 1997; Wu et al., 1997; United States Geological Survey, 1998; Wu et al., 1998a; Wu, 1999a). We shall first define the roles of these two systems under current development in Taiwan:

1. An RRS provides information (hypocenter, magnitude, and intensity) within about a minute or less after the event occurrence to populated areas and other sensitive locations. The RRS transmits this critical information quickly to emergency-response agencies. These agencies can then take action (some of which are preprogrammed) immediately after an earthquake has struck. The response measures can include the dispatch of rescue missions to the likely areas of damage.

2. An EWS forewarns an urban area of the forthcoming strong shaking, normally with a few seconds to a few tens of seconds of early-warning time. Even a few seconds of advanced-warning time will be useful for preprogrammed emergency measures, such as the deceleration of rapid-transit vehicles and high-speed trains to avoid potential derailment, the orderly shutoff of gas pipelines to minimize fire hazards, the controlled shutdown of manufacturing operations to reduce potential losses, and the safe-guarding of computer facilities to avoid the loss of vital databases.

The Taiwan Central Weather Bureau (CWB) has utilized its Rapid Earthquake Information Release System (RTD) since 1995 (Teng et al., 1997; Wu et al., 1997, 1998a, 2000) as a basis for the development of RRS and EWS capabilities. This RTD system (Fig. 1) uses a real-time, strong-motion accelerograph network that currently consists of 79 teleme- 
tered strong-motion stations distributed across Taiwan, an area of $100 \mathrm{~km}$ by $300 \mathrm{~km}$. Each station has three-component, force-balanced accelerometers with signals digitized at 50 samples per sec per channel at 16-bit resolution. The full recording dynamic range is $\pm 2 g$, and the sensitivity is sufficient to record $M>4.0$ events at a distance of approximately $100 \mathrm{~km}$. This sensitivity is desirable during the research development stage because we need to use the relatively frequent $M \sim 4$ events to test the system. For RRS and EWS operations, the real application is for large $(M>$ 6.5) events, for which all RTD stations throughout Taiwan will record the event clearly. Signals recorded on all conventional high-gain seismic networks for events of this size will be saturated and will therefore provide little of the needed information. By 1995, the RTD system had developed as one of its operating configurations an RRS system that was able to deliver reporting information within about 1 min after an earthquake occurrence (Teng et al., 1997; Wu et al., 1997, 2000). For the purpose of earthquake early

Taiwan Earthquake Rapid Information Release System (RTD)

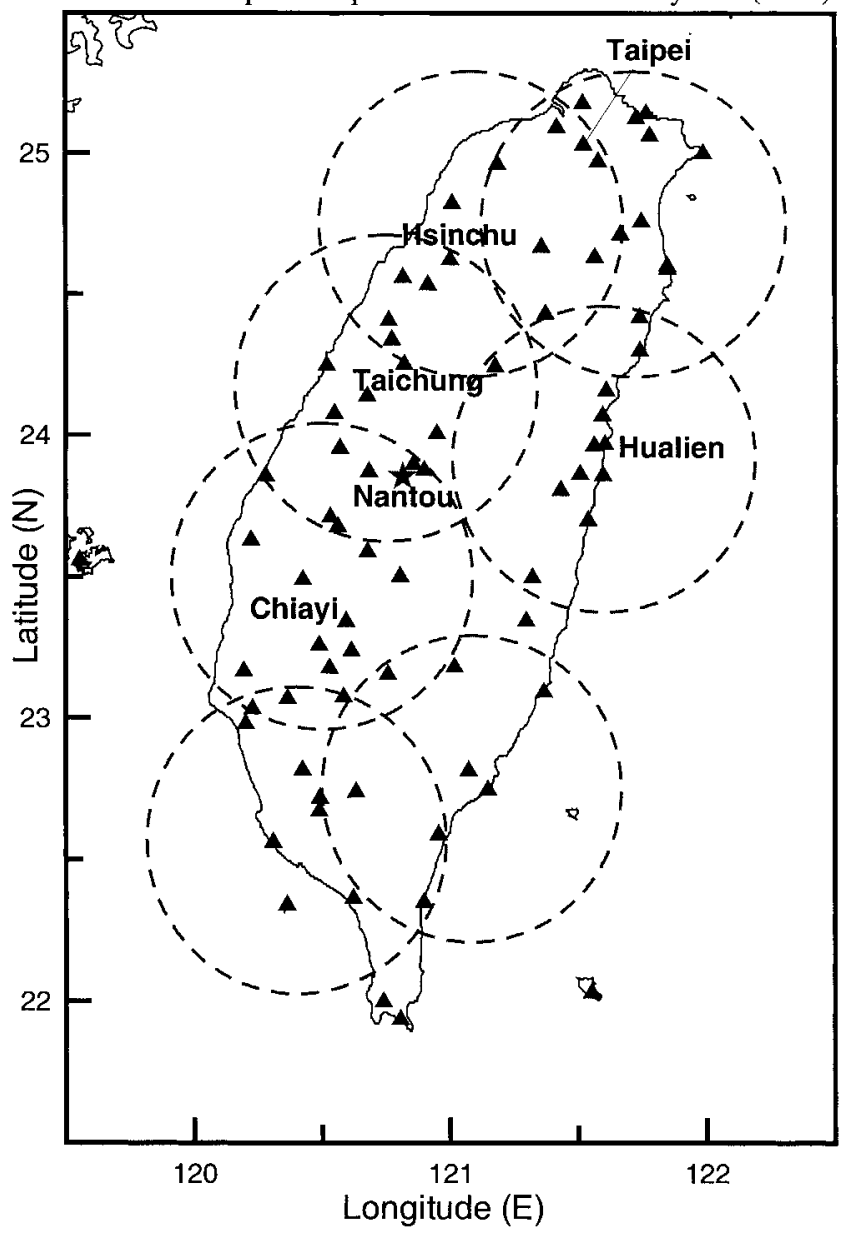

Figure 1. Station distribution of the RTD system and sample VSN networks of 60-km radius configured by software from the RTD system. Each VSN network is centered at a hypothetical event in Taiwan. The star shows the 1999 Chi-Chi mainshock epicenter. warning, this 1-min reporting time is too long. We have introduced a virtual subnetwork (VSN), a subset of RTD stations configured automatically by the monitoring software, to significantly shorten this reporting time. Results from a 6-month operation are presented in this article.

The reporting time $T r$ is determined by the time $T d$ needed for the system to trigger and record a sufficient length of the waveforms and the time $T p r$ needed to process the waveforms for the hypocenter and magnitude. The earthquake early-warning time $T w$ is given by:

$$
T w=T s-T r
$$

and

$$
T r=(T d+T p r),
$$

where $T s$ is the destructive $S$-wave travel time. For advanced warning, we must have $T w>0$. Clearly, this requires $T s>$ $(T d+T p r)$. Figure 2 gives a diagram of these times using results from one of the 54 events reported here. Sun Moon Lake $(\Delta=12 \mathrm{~km})$ is a VSN station in this case, and Taipei (a target area) is at $\Delta=143 \mathrm{~km}$. If $T d$ is allowed to be 15 $\mathrm{sec}$ (measured from the origin time) to complete the VSN triggering and recording and it takes $T p r=4 \mathrm{sec}$ to compute the hypocenter and magnitude, then we have an early-warning time $T w=23 \mathrm{sec}$ in Taipei. In this example, the waveform recorded at the Sun Moon Lake station provides excellent data for location and magnitude determinations. On the other hand, Figure 3 further illustrates these times using the 1999 Chi-Chi earthquake as an example. Consider two metropolitan areas: Taipei at $145 \mathrm{~km}$ away and Taichung at $35 \mathrm{~km}$

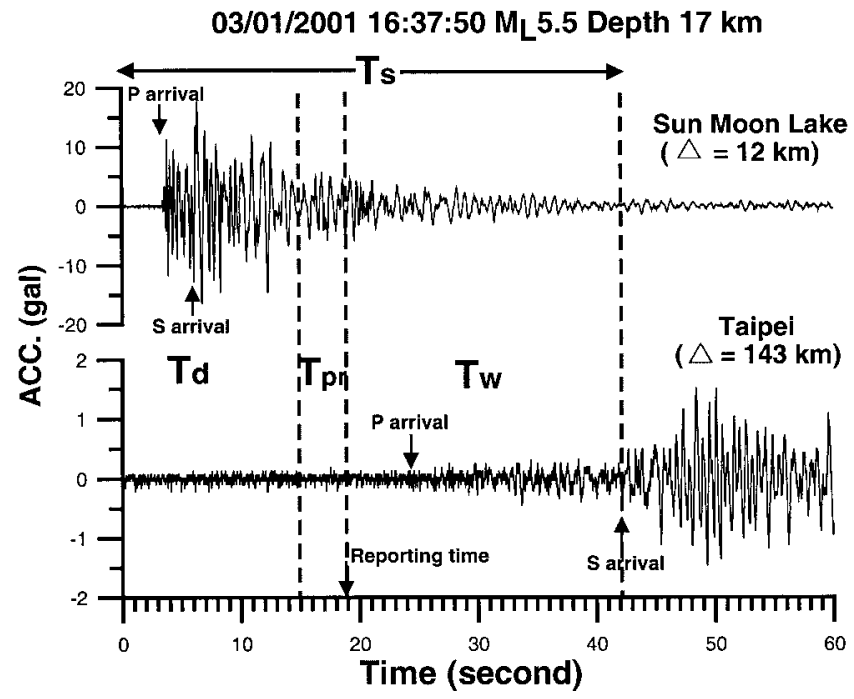

Figure 2. Diagram shows data-recording time $(T d)$, data-processing time (Tpr), and event-reporting time $(\mathrm{Tr})$ at a VSN station at Sun Moon Lake. Also shown are target-area early-warning time $(T w)$ and shear-wave travel time $(T s)$ of an earthquake. In this example, the target area Taipei (at $143 \mathrm{~km}$ away) has an earthquake early-warning time $T w=23 \mathrm{sec}$. 


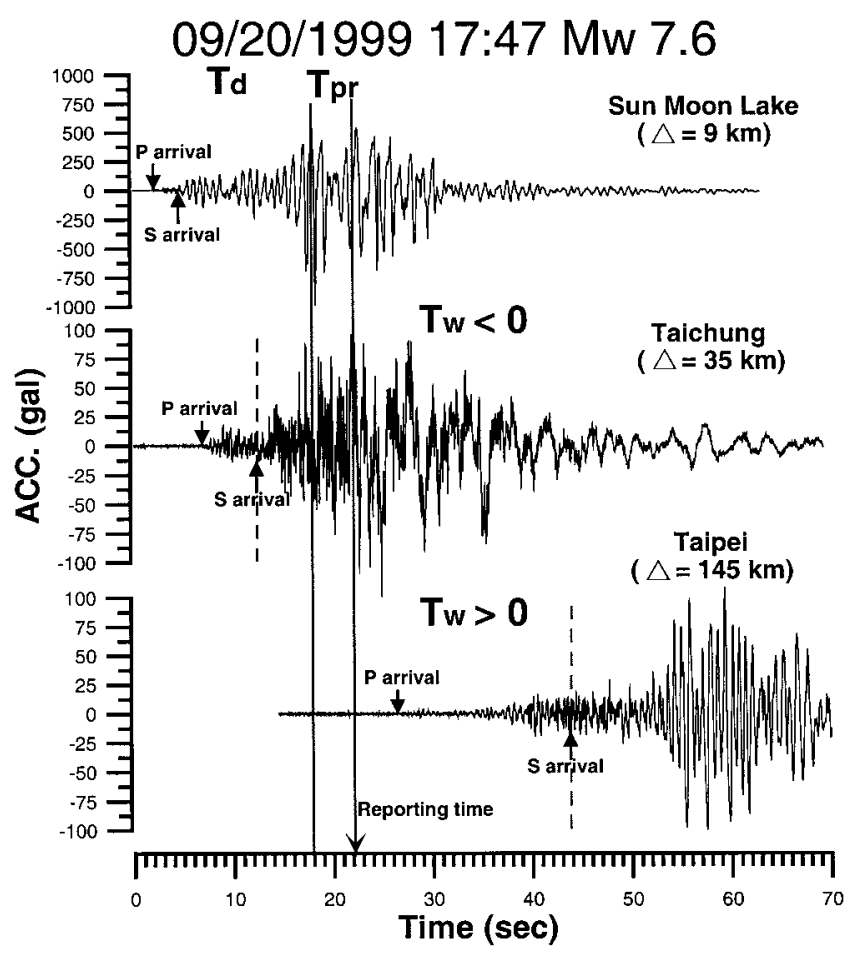

Figure 3. Illustration of the early-warning times for two target areas: Taipei at $145 \mathrm{~km}$ away and Taichung at $35 \mathrm{~km}$ away from the 1999 Chi-Chi mainshock. With a 22-sec reporting time, the strongshaking shear waves would have reached Taichung $10 \mathrm{sec}$ before the arrival of the EWS report. Thus $T w$ $<0$, and there is no early warning possible. In Taipei, $T w>0$, the larger epicentral distance allows a 22-sec early-warning time.

away. With a 22-sec reporting time $\operatorname{Tr}(T r=T d+T p r)$, the strong-shaking shear waves would have reached Taichung $10 \mathrm{sec}$ before the arrival of the EWS report. Thus, $T w<0$, and no early warning is possible. In Taipei, we have $\mathrm{Tw}>0$, for the larger epicentral distance allows a 22 -sec early-warning time, during which numerous preprogrammed measures can be taken to mitigate the hazard presented by the 100-gal shaking. In most cases, $T s$ cannot be much larger than $40 \mathrm{sec}$. This would correspond to an epicentral distance larger than about $160 \mathrm{~km}$, at which distance wave attenuation would have sufficiently reduced the impact of the strong shaking, except for special cases such as that of Mexico City (Espinosa-Aranda et al., 1995). Therefore, in general, $\mathrm{Tr}$ should be much less than $40 \mathrm{sec}$, and the shorter, the better. $T p r$ depends on the computer speed, the number of channels (or the size of the network) to be processed, and the algorithms used for hypocentral and magnitude determinations. $T d$ consists of the time needed for the system to (1) detect and confirm an event and (2) record a sufficient length of the waveforms so that a reliable hypocenter and magnitude can be determined. Our experience shows that part 1 of $T d$, determined by how fast the $P$-wave front sweeps across the network, normally takes about 1-3 sec for a small network, and part 2 of $T d$ takes a much longer time (usually $10 \mathrm{sec}$ or longer), as the system has to wait for the $S$ waves from remote stations to be recorded. Both $T p r$ and $T d$ are proportional to the size of the network. Clearly, the logical strategy is to shrink the size of the network without significantly sacrificing seismic coverage.

We have performed numerous experiments aimed at further reducing the computer processing time. The hypocenter-determination time can be reduced to about $0.1 \mathrm{sec}$ (Wu, 1999b), and the magnitude-determination time can be reduced to about the same order of magnitude. In an earlier experiment in Hualien, Taiwan, we have demonstrated that $T d$ can be significantly shortened by using a smaller network (Wu et al., 1997; Wu, 1999a; Wu et al., 1999). This leads to the design and configuration of a VSN within the hardware system of the ongoing RTD network. The VSN is event dependent and its configuration varies with time. By working with the VSN, we can substantially reduce the reporting time such that an earthquake early-warning capability is feasible. This article summarizes our research results and presents our findings.

\section{VSN System Development}

We have pointed out in the previous section that the large computer throughput caused by the large number of input channels $(79 \times 3)$ slows down the processing. Furthermore, to wait for the signals to come in from distant network stations consumes too much time, and these distant signals contribute only marginal information to the hypocenter and magnitude determinations. In actual hypocenter and magnitude determinations, only stations close to the epicenter (less than $60 \mathrm{~km}$ ) contribute crucial information. Within the framework of the RTD network, we choose to process signals only from a subset of the RTD stations surrounding an event. As soon as the RTD is triggered by an event, the system automatically extracts a subset of the RTD input-signal channels and configures a VSN with a $60-\mathrm{km}$ radius centered on that event. Figure 1 also gives a number of possible VSN configurations; each normally consists of about a dozen stations. The extracted data stream for this event forms the basic VSN input data for the subsequent RRS and EWS work.

\section{System Configuration}

Figure 4 shows the VSN system-hardware configuration. The $3 \times 79$ signal channels of the RTD system are telemetered from field stations to the CWB headquarters via digital telephone lines. The RTD system uses the conventional Short-Term-Average (STA)/Long-Term-Average (LTA) algorithm, with STA set at $0.3 \mathrm{sec}$ and LTA at $30 \mathrm{sec}$. When a station reaches the value of STA/LTA $>3.0$ and remains there for longer than $0.1 \mathrm{sec}$, this station is considered "triggered." The normal RTD recording goes on as more adjacent stations are triggered. An event is declared when the number of triggered stations reaches seven. At this time, two actions 


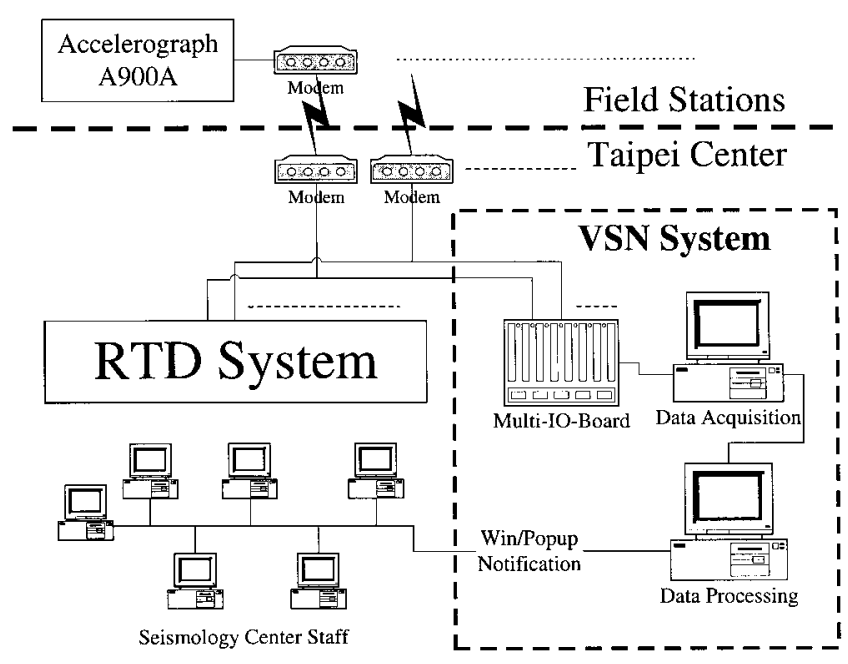

Figure 4. The RTD and VSN system-hardware configuration.

will be taken. First, the geometric center of these triggered stations is defined to be the "working" epicenter, and second, all RTD stations within $60 \mathrm{~km}$ of this working epicenter are defined to be the VSN, insofar as this declared event is concerned. Then the standby VSN computer will extract a copy of all signal traces of this VSN for early-warning data processing.

Signals of all stations within a $60-\mathrm{km}$ radius are grouped and extracted through a multi-input-output board to form the VSN input, which will then be processed in parallel through the VSN software in a dedicated computer. The time delay in signal telemetry is negligible, about a few milliseconds. This is obtained by bouncing a signal around the island. The delay in data logger, based on the size of the written files, is selected to be 3 sec.

We have conducted a series of experiments to determine the optimum recording time for a $60-\mathrm{km}$-radius network. Our results show that $10 \mathrm{sec}$ is about the optimum. As soon as the 10-sec waveforms are presented at the VSN system, they will be immediately processed to give simulated WoodAnderson seismograms for magnitude determinations. Figure 5a shows a sample of the VSN input, and Figure $5 \mathrm{~b}$ gives the corresponding simulated Wood-Anderson data stream. In this example, at 10 -sec recording time $T d$, only the top two stations have recorded sufficient lengths of $S$ waves to give adequate peak-displacement-amplitude measurements for local magnitudes (triangles in Fig. 5b), while other data channels give incorrectly low peak-displacement-amplitude readings compared with the RTD manual measurements (circles in Fig. 5b). Further reduction of this recording time will cause a significant reduction in the reliability and stability of the magnitude determinations, because insufficient lengths of large-amplitude $S$ waves are available. An increase of the recording time, on the other hand, will severely diminish the earthquake early-warning time $T w$ without a significant improvement in magnitude determination. The
VSN system is programmed to continue the recording of the waveforms up to $10 \mathrm{sec}$ after the first $P$ arrival, then hypocenter and magnitude determinations will be carried out. Results are disseminated automatically to users through a Win/ Popup Notification software.

\section{Hypocenter Determination}

Based on the conventional STA/LTA algorithm, the automatic phase-picking technique (Wu et al., 1998b) is used to first pick $P$-wave arrivals from the vertical components during the formation of the VSN. When the $P$-wave arrivals are picked and the event is confirmed, the system will pick the $S$-wave arrivals from the two horizontal components. After the automatic phase-picking process is completed, the hypocenter-determination program is run using a 1-D continuous-velocity model. The hypocenter location produced by the program is the one that best matches the observed arrival times predicted by the 1-D continuousvelocity model with minimized root-mean-square traveltime residuals.

We have long used a layered-earth model in our RTD system. However, the computed root-mean-square based on a layered-earth model normally produces more than one local minimum in addition to the global minimum we seek. It takes time to sort out whether the first trial hypocenter location is trapped within the "valley" of a certain local minimum to which the solution converges. Using a 1-D continuous-velocity model can avoid such problems: there is only one global minimum. We thus incorporate this program into the VSN system to avoid spending extra time in seeking the global minimum among many local minima. The normal running time for hypocenter determination is about $0.1 \mathrm{sec}$ (Wu, 1999b).

The hypocenter location produced by the 1-D continuous-velocity model may not be exactly the same as the RTD hypocenter, but it is generally close enough for the practical purpose of early warning. Speed and stability are the two merits of the 1-D continuous-velocity model earthquakelocation program, and they are very important for an earlywarning system. The small error (few $\mathrm{km}$ ) introduced is indeed insignificant in earthquake early warning, which is intended for large events of tens to hundreds of kilometers in source dimension. After all, the hypocenter determined by the first $P$ and $S$ arrivals is itself an approximation, which gives only the rupture nucleation point.

\section{Magnitude Determination}

A major task in developing the VSN system is the nearly real-time estimate of the earthquake magnitude. Since both the RRS and the EWS are mainly intended for earthquakes larger than magnitude 6.5, moment magnitude is the appropriate measurement. For real-time seismic monitoring, we have developed a recursive moment-tensor-inversion algorithm (Qu and Teng, 1994) through which the moment magnitude can be derived. However, we have not yet been able to decrease the computational speed to meet the EWS re- 
(a)

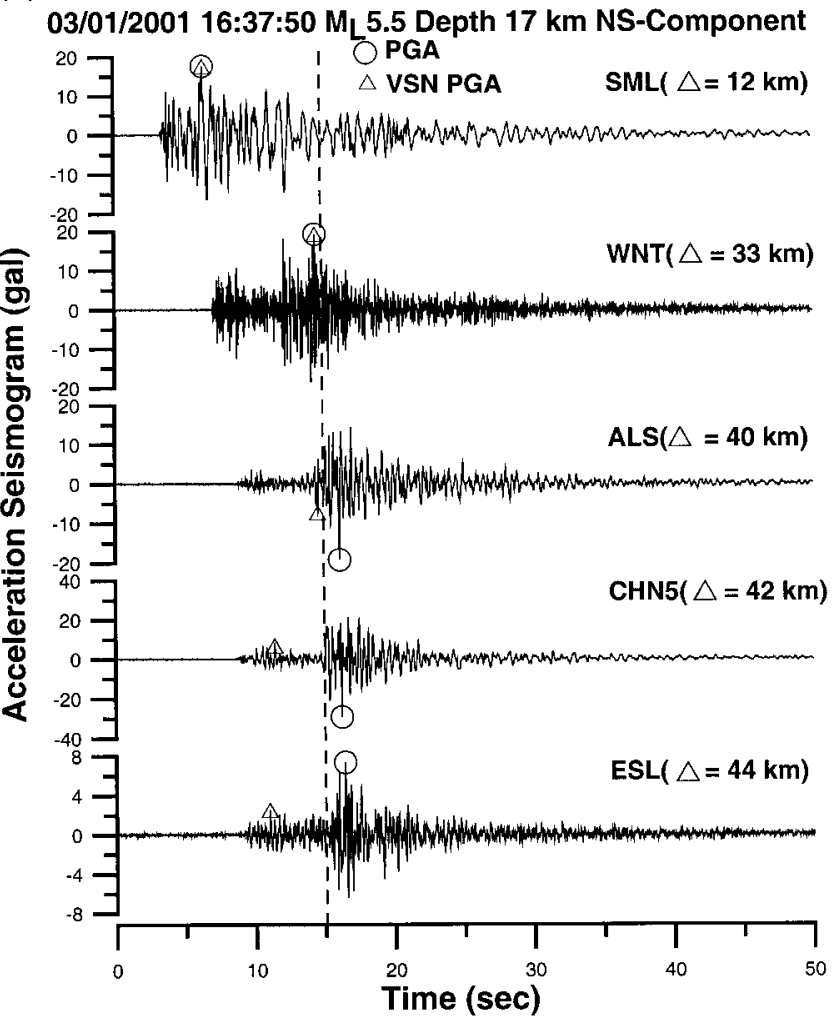

(b)

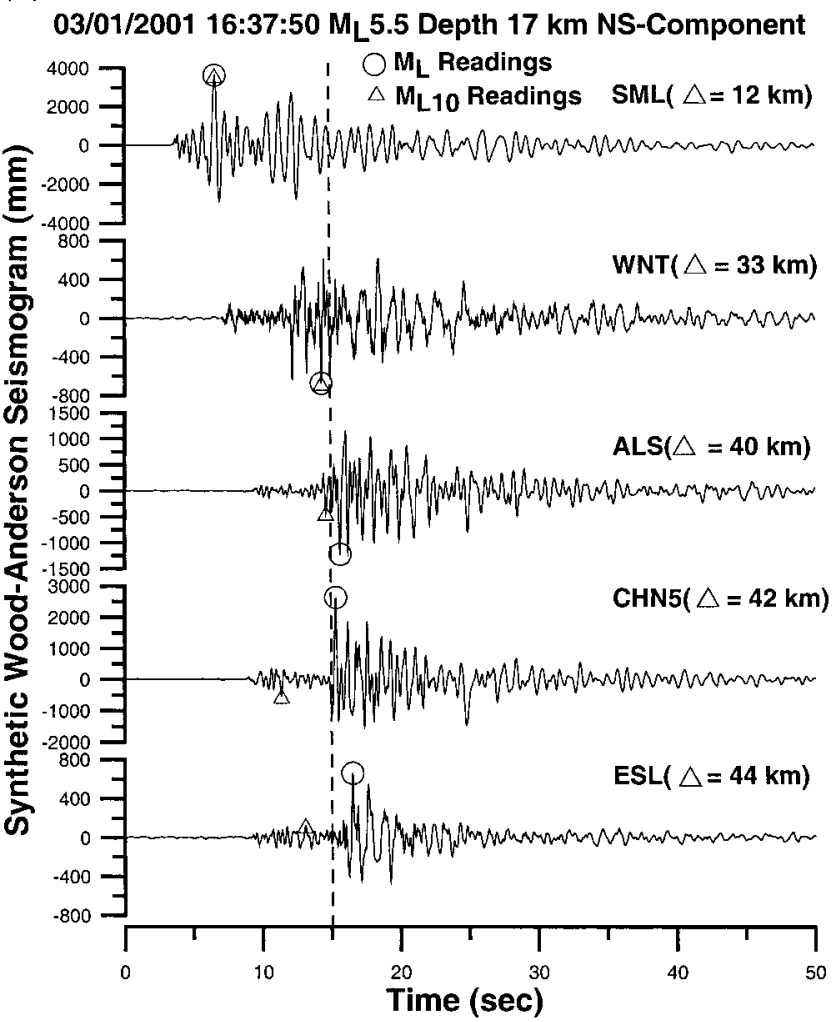

Figure 5. (a) A sample VSN acceleration data stream. (b) The corresponding simulated Wood-Anderson data stream from which $M_{\mathrm{L} 10}$ is measured. A circle gives the offline manual peak-amplitude measurement; a triangle gives the on-line real-time peakamplitude measurement within the 10 -sec window.

quirement. We currently have designed an empirical approach, also based on a recursive scheme, to help deduce the moment magnitude in real time:

1. Once an event is triggered, the entire VSN data stream will be recorded for $10 \mathrm{sec}$ after the first $P$ arrival. A number of stations in the VSN will have recorded both the $P$ and the $S$ waveforms, while other stations may have recorded only partial $S$ waveforms (Fig. 5a).

2. As signals are coming in, a recursive filter developed by Kanamori et al. (1999) is applied to the horizontal components of the accelerograms to give simulated WoodAnderson seismograms (Fig. 5b). As soon as the hypocenter is obtained, epicentral distances to each VSN station are known. Maximum amplitudes measured from the Wood-Anderson seismograms, together with the known distance corrections, will give an average local magnitude, $M_{\mathrm{L} 10}$, measured with the 10 -sec waveforms among the VSN stations. Using this $M_{\mathrm{L} 10}$ measurement, we can get $M_{\mathrm{L}}$ by an empirical relation based on the RTD data CWB seismic network has obtained earlier (Wu et al., 1998a):

$$
M_{\mathrm{L}}=1.28 \times M_{\mathrm{L} 10}-0.85
$$

This relation, obtained from a previous database, allows the conversion of the local-magnitude value derived from the 10-sec VSN waveforms $\left(M_{\mathrm{L} 10}\right)$ to an $M_{\mathrm{L}}$ value determined off-line by the conventional manner.

3. From $M_{\mathrm{L}}$, another empirical relation derived from past $M_{\mathrm{L}}$ and $M_{\mathrm{W}}$ data gives the moment magnitude $M_{\mathrm{W}}$ (Fig. 6) as described in our previous study (Wu et al., 2001):

$$
M_{\mathrm{L}}=4.533 \times \ln \left(M_{\mathrm{w}}\right)-2.091
$$

This empirical relation is based on Taiwan events with $M_{\mathrm{L}}$ determined manually off-line and $M_{\mathrm{W}}$ taken from the Harvard catalog. It allows the conversion of $M_{\mathrm{L}}$ to $M_{\mathrm{W}}$.

Since the recursive filtering is simple and the transformations through the two empirical relations (1) and (2) are trivial, magnitude determination can be accomplished within a fraction of a second after the completion of the 10-sec VSN recording. Admittedly, the saturation problem of the $M_{\mathrm{L}}$ measurement for large events remains to be resolved. The real-time recursive moment-tensor inversion is the ultimate solution to the problem of getting a reliable real-time $M_{\mathrm{W}}$ measurement. For the test events of magnitude smaller than 6 as presented in this article, the present scheme used is quite adequate. 


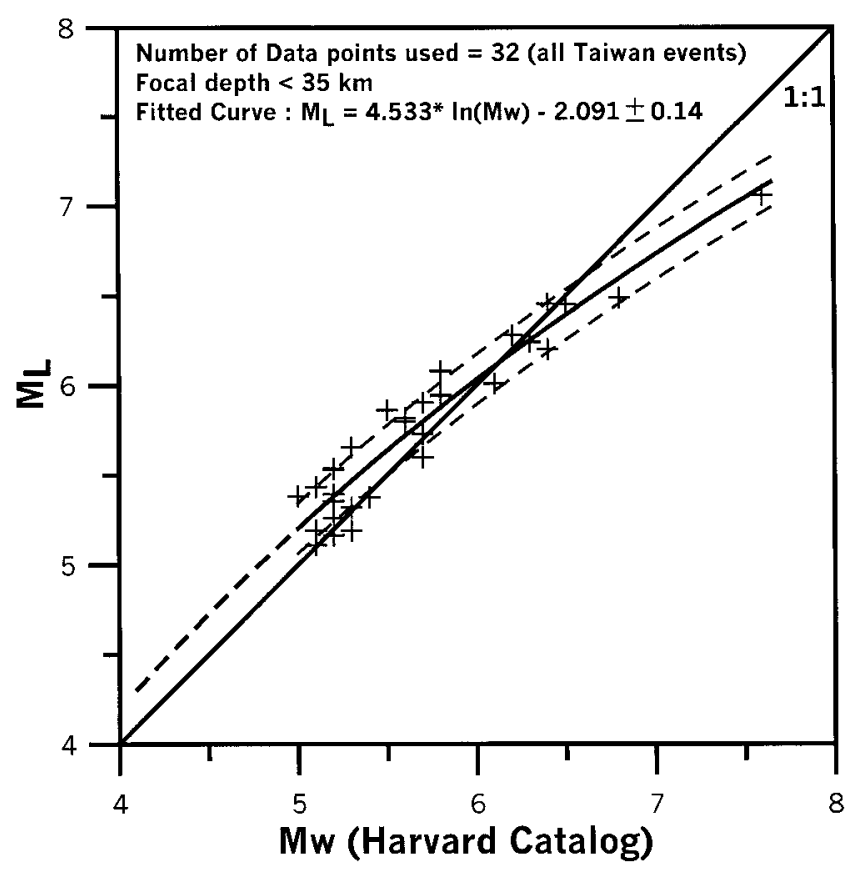

Figure 6. Relation between $M_{\mathrm{W}}$ and $M_{\mathrm{L}}$ based on shallow-earthquake data in Taiwan region.

\section{Results}

We have implemented the aforementioned VSN operation on the existing RTD network during the period from December 2000 to June 2001. A total of 54 earthquakes of magnitude $M_{\mathrm{L}}$ ranging from 3.5 to 6.3 were detected, realtime processed, and reported. The system correctly detected and reported all events. The performance of the VSN system is summarized in Table 1, which lists the hypocenter locations, local magnitudes, moment magnitude, and reporting times $\operatorname{Tr}$ for all 54 events. As a comparison, the VSN automatic on-line results are presented in parallel with the RTD results determined manually off-line. Analyses of these results are given in the next paragraphs.

\section{Location}

Hypocenters from the VSN automatic real-time processing versus those from the RTD system manual-off-line processing are plotted in Figure 7. Hypocenters determined by the two systems are generally in good agreement except for a few events that show large differences. The average difference in epicenter location is $4.2 \mathrm{~km}$ with a standard deviation of $7.3 \mathrm{~km}$, and the average difference in focal depth is $4.5 \mathrm{~km}$ with a standard deviation of $5.5 \mathrm{~km}$. The average difference for the epicenter is about the same as that for the focal depth. The standard deviation for the epicenter is larger than that for the focal depth. This is due to a small number of events that have large differences in epicenters given by the two systems. Because the VSN system uses shortened waveforms and a much smaller number of stations, occa- sional large location errors in the VSN system are expected due to a reduction of station coverage and missing $S$-arrival times. However, the quality of the locations produced by the VSN system is sufficiently good for RRS and EWS applications, as they are mainly intended for large events where the source dimension can be an order of magnitude larger than the location errors. Moreover, the hypocenter determined by the first $P$ and $S$ arrivals generally represents the nucleation point of the ensuing rupture, which may depart from the location of the principal asperity where the greatest energy release takes place. With these considerations, we think that the VSN hypocenter determination is adequate for the intended purposes.

\section{Magnitude}

The real-time magnitude-determination process described previously has been applied in real time to the partial waveforms (10 sec after the first $P$ arrival), and the magnitudes $M_{\mathrm{L} 10}$ are obtained accordingly. They are listed in Table 1 under the VSN columns. The corresponding $M_{\mathrm{L}}$ values determined manually off-line are also listed in Table 1 under the RTD columns. As expected, $M_{\mathrm{L} 10}<M_{\mathrm{L}}$, because shorter waveforms are used in the VSN system. We can get the corresponding local-magnitude values through a correction by using equation (1). They are listed in Table 1 as $M_{\mathrm{L} 10 \mathrm{C}}$, which are the magnitude values reported in this EWS experiment. It is interesting to check how the actual data from these 54 events would modify equation (1). Figure 8 give a plot of the $M_{\mathrm{L} 10}$ values against the off-line, manually determined $M_{\mathrm{L}}$ values from the RTD system. A regression line gives

$$
M_{\mathrm{L}}=1.39 \times M_{\mathrm{L} 10}-1.49,
$$

which is slightly different from the plot of equation (1), shown as the thick, dashed line in Figure 8. For large earthquakes, these two lines are essentially the same. Using either equation (1) or (3), the magnitude uncertainty is on the order of \pm 0.25 , a reasonable value that is smaller than Grecksch and Kumpel (1997) obtained in their magnitude determination for EWS. They estimated magnitudes using the rise time of the first complete $P$-wave cycle, the predominant period, and the related Fourier amplitude of the initial part of strongmotion signals. However, with this approach, the magnitude uncertainty can be as much as \pm 1.35 from a single accelerogram and \pm 0.5 from averaging data from more than eight accelerograms. Other researchers (e.g., Nakamura, 1988, 1989; Tsai and $\mathrm{Wu}, 1997$ ) have also tried to estimate magnitude from the initial portion of accelerograms, but large uncertainties are still a major problem. The uncertainty becomes severe for a large rupture with an extended nucleation process.

As the real-time recursive moment-tensor-inversion procedure has not yet been successfully implemented, a shortcut would be to employ an empirical relation (equation 
Table 1

Results of Earthquake Early-Warning Research

\begin{tabular}{|c|c|c|c|c|c|c|c|c|c|c|c|c|c|}
\hline \multirow[b]{2}{*}{ Date } & \multirow[b]{2}{*}{ Origin Time } & \multicolumn{4}{|c|}{ RTD Offline (Manual) } & \multicolumn{6}{|c|}{ VSN Online (Automatic) } & \multirow{2}{*}{$\begin{array}{l}\text { Reporting } \\
\text { Time }\end{array}$} & \multirow[b]{2}{*}{$\operatorname{Tr}(\mathrm{sec})$} \\
\hline & & Lat. $(\mathrm{N})$ & Lon. (E) & Dept h (km) & $M_{\mathrm{L}}$ & Lat. (N) & Lon. (E) & Dept h (km) & $M_{\mathrm{L} 10} \mathrm{c}$ & $M_{\mathrm{L} 10}$ & $M_{\mathrm{W}}$ & & \\
\hline $10 / 12 / 00$ & 10:08:39 & 23.11 & 120.2 & 19 & 5.1 & 23.14 & 120.23 & 16 & 5.3 & 4.8 & 5.1 & 10:09:10 & 31 \\
\hline $10 / 12 / 00$ & 20:00:20 & 23.06 & 120.1 & 21 & 4.7 & 23.05 & 120.15 & 20 & 4.6 & 4.3 & 4.4 & 20:00:43 & 23 \\
\hline $10 / 12 / 00$ & $21: 34: 18$ & 23.06 & 120.1 & 21 & 4.5 & 23.06 & 120.18 & 19 & 4.9 & 4.5 & 4.7 & $21: 34: 59$ & 41 \\
\hline $20 / 12 / 00$ & $01: 22: 23$ & 24.09 & 121.5 & 21 & 4.5 & 24.09 & 121.52 & 18 & 4.8 & 4.4 & 4.5 & $01: 22: 42$ & 19 \\
\hline $22 / 12 / 00$ & $11: 22: 54$ & 24.06 & 121.1 & 4 & 4.8 & 24.08 & 121.13 & 3 & 4.8 & 4.4 & 4.6 & $11: 23: 18$ & 24 \\
\hline $28 / 12 / 00$ & 09:01:29 & 23.48 & 121.8 & 11 & 5.1 & 23.58 & 121.78 & 32 & 5.7 & 5.1 & 5.5 & 09:02:00 & 31 \\
\hline 04/01/01 & $19: 03: 38$ & 24.73 & 121.7 & 11 & 4.1 & 24.70 & 121.77 & 13 & 4.2 & 4.0 & 4.0 & $19: 03: 54$ & 16 \\
\hline 09/01/01 & $19: 16: 29$ & 24.03 & 121.7 & 28 & 5.1 & 24.01 & 121.64 & 40 & 5.2 & 4.7 & 5.0 & $19: 16: 46$ & 17 \\
\hline 09/01/01 & $19: 38: 32$ & 24.03 & 121.7 & 30 & 4.7 & 24.01 & 121.69 & 40 & 4.9 & 4.5 & 4.7 & $19: 38: 50$ & 18 \\
\hline $11 / 01 / 01$ & 08:37:00 & 24.06 & 120.9 & 23 & 5.1 & 24.07 & 120.99 & 13 & 5.0 & 4.5 & 4.7 & $08: 37: 22$ & 23 \\
\hline $11 / 01 / 01$ & 09:09:11 & 24.08 & 120.9 & 26 & 4.9 & 24.02 & 120.99 & 9 & 4.3 & 4.0 & 4.1 & 09:09:29 & 18 \\
\hline $16 / 01 / 01$ & 01:43:07 & 24.26 & 121.1 & 17 & 4.9 & 24.26 & 121.18 & 16 & 5.1 & 4.6 & 4.9 & $01: 43: 30$ & 23 \\
\hline $22 / 01 / 01$ & $23: 27: 20$ & 24.35 & 122.0 & 18 & 5.3 & 24.37 & 122.07 & 18 & 5.7 & 5.1 & 5.6 & $23: 27: 53$ & 33 \\
\hline $24 / 01 / 01$ & $22: 10: 10$ & 23.58 & 121.4 & 39 & 4.4 & 23.85 & 121.09 & 21 & 4.7 & 4.3 & 4.5 & $22: 10: 36$ & 26 \\
\hline $28 / 01 / 01$ & 13:09:37 & 23.46 & 120.4 & 11 & 4.3 & 23.46 & 120.42 & 12 & 4.4 & 4.1 & 4.2 & $13: 09: 53$ & 16 \\
\hline 04/02/01 & $23: 54: 15$ & 24.27 & 121.6 & 16 & 4.3 & 24.28 & 121.69 & 3 & 4.3 & 4.0 & 4.1 & $23: 54: 35$ & 20 \\
\hline $05 / 02 / 01$ & $08: 37: 34$ & 24.27 & 121.7 & 12 & 4.3 & 24.26 & 121.69 & 16 & 4.8 & 4.4 & 4.6 & $08: 37: 57$ & 23 \\
\hline $05 / 02 / 01$ & $08: 39: 38$ & 24.25 & 121.7 & 17 & 3.9 & 24.25 & 121.69 & 16 & 4.1 & 3.9 & 3.9 & 08:40:07 & 29 \\
\hline 08/02/01 & $17: 34: 10$ & 23.42 & 120.6 & 13 & 4.8 & 23.42 & 120.64 & 13 & 4.7 & 4.4 & 4.5 & $17: 34: 30$ & 20 \\
\hline 08/02/01 & $18: 17: 18$ & 23.42 & 120.6 & 15 & 4.1 & 23.42 & 120.63 & 13 & 4.2 & 3.9 & 4.0 & $18: 17: 35$ & 16 \\
\hline $11 / 02 / 01$ & $12: 43: 26$ & 23.93 & 120.7 & 16 & 4.5 & 23.92 & 120.74 & 15 & 4.9 & 4.5 & 4.7 & $12: 43: 51$ & 25 \\
\hline $14 / 02 / 01$ & $13: 17: 50$ & 23.59 & 120.7 & 19 & 4.3 & 23.59 & 120.73 & 17 & 4.4 & 4.1 & 4.2 & $13: 18: 08$ & 18 \\
\hline $14 / 02 / 01$ & $22: 25: 43$ & 23.59 & 120.7 & 16 & 4.6 & 23.58 & 120.75 & 14 & 4.3 & 4.0 & 4.1 & $22: 25: 58$ & 15 \\
\hline $18 / 02 / 01$ & $10: 31: 46$ & 23.57 & 120.6 & 11 & 4.2 & 23.58 & 120.68 & 15 & 4.4 & 4.1 & 4.2 & $10: 32: 02$ & 16 \\
\hline $18 / 02 / 01$ & $20: 25: 11$ & 23.59 & 120.7 & 17 & 5.1 & 23.58 & 120.74 & 16 & 5.1 & 4.7 & 4.9 & $20: 25: 33$ & 22 \\
\hline $20 / 02 / 01$ & $01: 41: 22$ & 23.59 & 120.7 & 18 & 4.3 & 23.60 & 120.72 & 16 & 4.5 & 4.2 & 4.3 & $01: 41: 43$ & 21 \\
\hline $23 / 02 / 01$ & $19: 18: 28$ & 23.54 & 121.6 & 35 & 4.9 & 23.56 & 121.56 & 37 & 4.8 & 4.4 & 4.6 & $19: 18: 47$ & 19 \\
\hline 01/03/01 & $16: 37: 50$ & 23.84 & 121.0 & 17 & 5.5 & 23.83 & 121.00 & 20 & 5.3 & 4.8 & 5.1 & $16: 38: 09$ & 19 \\
\hline $04 / 03 / 01$ & 03:06:13 & 23.96 & 121.0 & 14 & 4.0 & 23.98 & 121.08 & 3 & 4.2 & 4.0 & 4.0 & 03:06:38 & 25 \\
\hline $11 / 03 / 01$ & $18: 24: 07$ & 23.59 & 120.7 & 21 & 4.6 & 23.60 & 120.74 & 19 & 4.8 & 4.4 & 4.6 & $18: 24: 25$ & 18 \\
\hline $12 / 03 / 01$ & $01: 47: 54$ & 23.59 & 120.7 & 16 & 4.4 & 23.58 & 120.74 & 20 & 4.6 & 4.2 & 4.3 & $01: 48: 16$ & 22 \\
\hline $12 / 03 / 01$ & $09: 28: 37$ & 23.23 & 120.4 & 15 & 3.9 & 23.17 & 120.46 & 16 & 4.3 & 4.1 & 4.1 & 09:29:04 & 27 \\
\hline $13 / 03 / 01$ & $22: 54: 29$ & 23.68 & 120.7 & 19 & 4.2 & 23.68 & 120.78 & 18 & 4.5 & 4.2 & 4.3 & $22: 54: 57$ & 28 \\
\hline $19 / 03 / 01$ & $02: 56: 58$ & 23.65 & 120.6 & 13 & 3.6 & 23.62 & 120.59 & 10 & 4.2 & 4.0 & 4.0 & $02: 57: 21$ & 23 \\
\hline $23 / 03 / 01$ & 01:03:17 & 22.84 & 121.2 & 27 & 4.5 & 22.80 & 121.27 & 33 & 5.1 & 4.6 & 4.8 & 01:03:39 & 22 \\
\hline 03/04/01 & 12:31:09 & 24.05 & 121.4 & 13 & 4.0 & 24.06 & 121.46 & 12 & 4.3 & 4 & 4.1 & $12: 31: 32$ & 23 \\
\hline 06/04/01 & $20: 22: 31$ & 23.95 & 121.4 & 20 & 3.6 & 23.93 & 121.41 & 16 & 3.8 & 3.7 & 3.7 & $20: 22: 50$ & 19 \\
\hline $21 / 04 / 01$ & $18: 52: 20$ & 23.31 & 120.7 & 16 & 4.0 & 23.34 & 120.72 & 3 & 3.9 & 3.7 & 3.8 & $18: 52: 38$ & 18 \\
\hline $24 / 04 / 01$ & 10:08:49 & 23.47 & 120.4 & 16 & 4.0 & 23.46 & 120.46 & 14 & 4.1 & 3.9 & 3.9 & 10:09:04 & 15 \\
\hline $24 / 04 / 01$ & $14: 24: 50$ & 23.81 & 120.9 & 12 & 4.1 & 23.81 & 120.97 & 13 & 4.1 & 3.9 & 3.9 & $14: 25: 06$ & 16 \\
\hline $27 / 04 / 01$ & $02: 02: 33$ & 23.66 & 121.0 & 7 & 5.1 & 23.64 & 121.04 & 5 & 4.9 & 4.5 & 4.7 & $02: 02: 55$ & 22 \\
\hline $27 / 04 / 01$ & $02: 06: 50$ & 23.62 & 121.0 & 3 & 4.0 & 23.63 & 121.05 & 4 & 4.3 & 4.0 & 4.1 & $02: 07: 20$ & 30 \\
\hline $29 / 04 / 01$ & $06: 37: 13$ & 23.59 & 120.7 & 14 & 4.4 & 23.55 & 120.78 & 16 & 4.9 & 4.5 & 4.6 & $06: 37: 31$ & 18 \\
\hline $30 / 04 / 01$ & $15: 35: 30$ & 23.98 & 121.0 & 16 & 3.9 & 23.99 & 121.01 & 16 & 3.9 & 3.7 & 3.8 & $15: 35: 44$ & 14 \\
\hline $01 / 05 / 01$ & $21: 55: 59$ & 24.25 & 121.1 & 6 & 4.2 & 24.23 & 121.09 & 3 & 4.4 & 4.1 & 4.1 & $21: 56: 21$ & 22 \\
\hline 08/05/01 & $18: 54: 15$ & 23.34 & 121.6 & 29 & 4.6 & 23.37 & 121.48 & 53 & 4.6 & 4.3 & 4.4 & $18: 54: 35$ & 20 \\
\hline $10 / 05 / 01$ & $04: 06: 36$ & 24.01 & 121.5 & 28 & 4.6 & 24.00 & 121.67 & 22 & 5.0 & 4.5 & 4.7 & $04: 06: 52$ & 16 \\
\hline $13 / 05 / 01$ & $14: 57: 04$ & 23.93 & 121.0 & 10 & 4.8 & 23.94 & 121.03 & 7 & 4.5 & 4.2 & 4.3 & $14: 57: 23$ & 19 \\
\hline $13 / 05 / 01$ & $23: 38: 06$ & 24.44 & 121.8 & 21 & 4.3 & 24.44 & 121.86 & 20 & 4.6 & 4.3 & 4.4 & $23: 38: 29$ & 23 \\
\hline $23 / 05 / 01$ & $19: 19: 58$ & 24.72 & 121.8 & 10 & 3.8 & 24.74 & 121.83 & 13 & 4.1 & 3.9 & 4 & $19: 20: 21$ & 23 \\
\hline $23 / 05 / 01$ & $19: 22: 05$ & 24.69 & 121.7 & 8 & 3.5 & 24.73 & 121.82 & 12 & 3.7 & 3.6 & 3.6 & $19: 22: 30$ & 25 \\
\hline $30 / 05 / 01$ & $22: 36: 53$ & 23.86 & 121.0 & 16 & 4.2 & 23.87 & 121.05 & 13 & 4.4 & 4.1 & 4.2 & $22: 37: 17$ & 24 \\
\hline $06 / 06 / 01$ & $09: 26: 32$ & 24.40 & 121.7 & 15 & 4.2 & 24.41 & 121.77 & 13 & 4.6 & 4.2 & 4.4 & $09: 26: 52$ & 20 \\
\hline $14 / 06 / 01$ & $02: 35: 26$ & 24.45 & 121.9 & 16 & 6.3 & 24.45 & 121.89 & 18 & 6.5 & 5.7 & 6.6 & $02: 35: 55$ & 29 \\
\hline
\end{tabular}




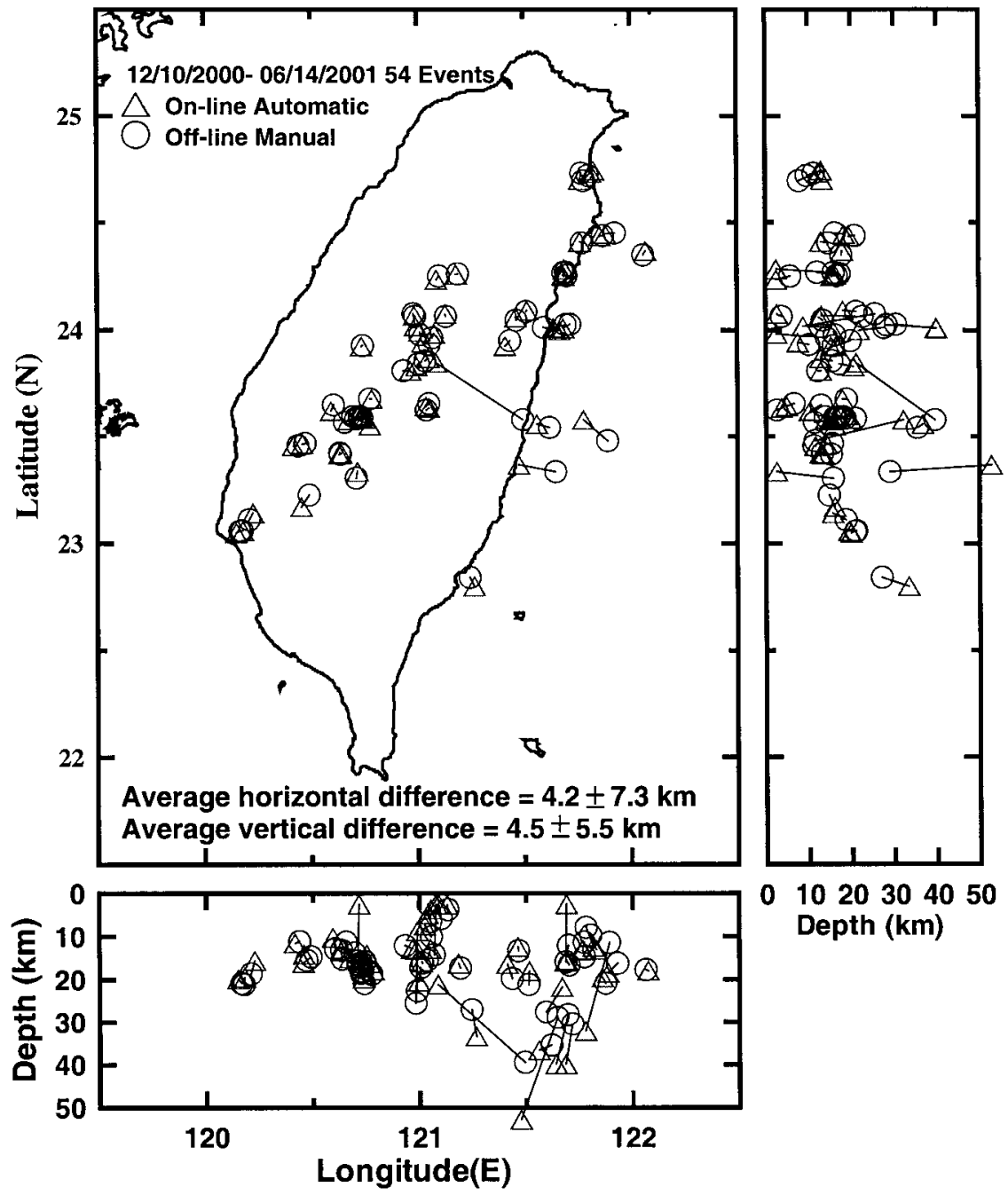

Figure 7. Comparison of the real-time automatic event locations of the VSN system and the manual off-line event locations of the RTD system.

2) between $M_{\mathrm{L}}$ and $M_{\mathrm{W}}$ as discussed before. We will use this relation for the time being to obtain the estimated $M_{\mathrm{W}}$ values from measured $M_{\mathrm{L}}$ values, knowing that the latter has magnitude-saturation problem for large events. For a very large earthquake, such as the Chi-Chi event, the source can be very complex, and it may be composed of multiple ruptures beginning with a small nucleation event. The accurate magnitude determination is nontrivial. The nucleation event may not be very big. As the VSN system makes magnitude determination based on the 10-sec VSN waveforms after the first declared $P$ arrival, a smaller magnitude (e.g., $M_{\mathrm{W}} 6.5$ ) might have been determined for the Chi-Chi event while the rupture was still going on. The system will issue an earlywarning message with an underestimated magnitude. We are currently configuring a multiparallel processing system, which will update the magnitude as rupture goes on. Results on this development will be reported later.
Early-Warning Time

With a 7-month operation of the VSN system, we have accomplished an earthquake-reporting time $\operatorname{Tr}$ that varies from 14 to $41 \mathrm{sec}$ for the 54 events (listed in the last column of Table 1), with an average of $21.9 \mathrm{sec}$ and a standard deviation of $5.3 \mathrm{sec}$ (Fig. 9). The result shows that for most events, the VSN system has delivered an early-warning report within $30 \mathrm{sec}$. Only four events have $\operatorname{Tr}$ longer than $30 \mathrm{sec}$, and three of them occurred in the early stages of the VSN operation, when parameters were being adjusted to fine-tune the system. A couple of these came from offshore, where a longer recording time is needed even for the $P$ waves to reach the VSN stations. By and large, the $\operatorname{Tr}$ for most of the events is short enough (about $22 \mathrm{sec}$ ) that a practical earthquake early warning can be accomplished. 


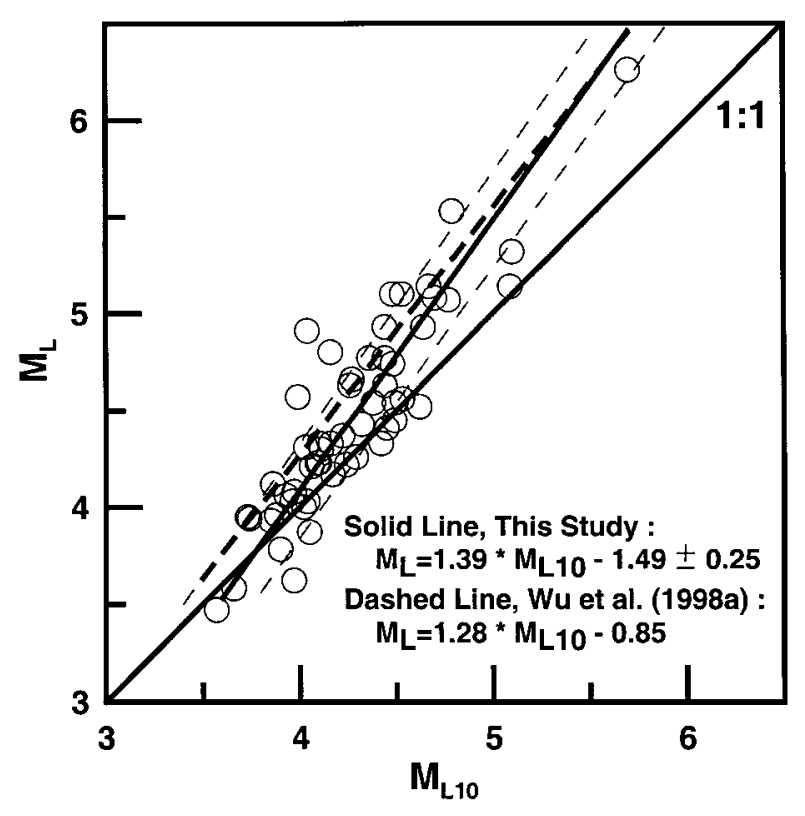

Figure 8. Correlations between $M_{\mathrm{L}}$ and $M_{\mathrm{L} 10}$ between this study and an earlier study by $\mathrm{Wu}$ et al. (1998a).

\section{Conclusions}

We have configured a VSN within the ongoing realtime, strong-motion, telemetered network RTD of the CWB of Taiwan. A 7-month continual operation shows that the VSN has a $100 \%$ correct-detection performance for a total of 54 larger events. The reporting time $\operatorname{Tr}$ is $30 \mathrm{sec}$ or less, with an average of about $22 \mathrm{sec}$. The 22 -sec reporting time of the VSN system will offer earthquake early warning for metropolitan areas located more than $100 \mathrm{~km}$ from the epicenter. For an event with the same location as the $20 \mathrm{Sep}-$ tember 1999 Chi-Chi, Taiwan, earthquake, the Taipei metropolitan area at $145 \mathrm{~km}$ would have more than $20 \mathrm{sec}$ of early-warning time as indicated by Figure 3. Figure 10 shows the expected early-warning times for an event like the Chi-Chi earthquake for all parts of Taiwan.

This result of a 22 -sec reporting time represents encouraging progress toward an earthquake early-warning capability, with early-warning time $T w$ ranging from a few seconds to a few tens of seconds for areas $75 \mathrm{~km}$ or farther from the epicenter. To minimize the earthquake-reporting time $\operatorname{Tr}$ (and to maximize the early-warning time $T w$ ), we have to limit the recording time $T d$ to about $10 \mathrm{sec}$ from the first $P$ arrival for a VSN network. This means we can only use a short initial section of the waveforms to derive all needed earthquake information. The configuration of a VSN in an operating high-density, telemetered, strong-motion network seems to give the desired answer.

The use of shorter waveforms in real time determinations of hypocenter and magnitude introduces errors. If we

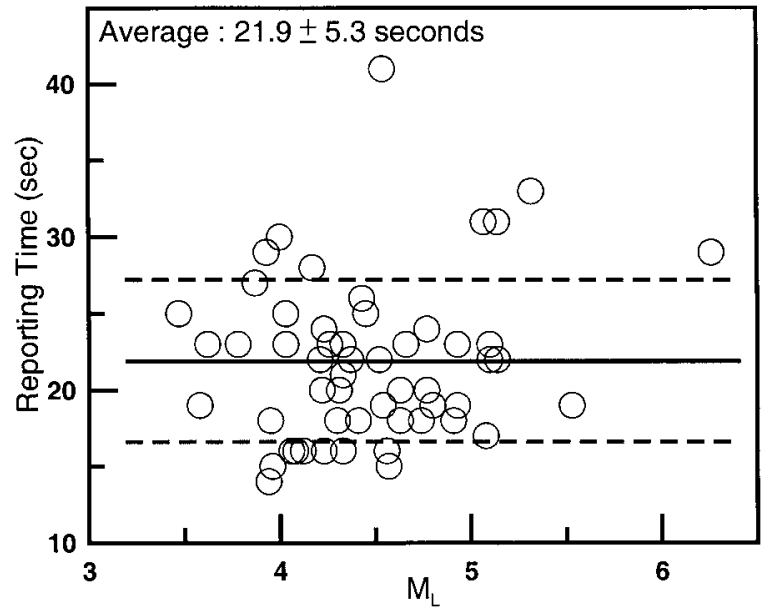

Figure 9. Earthquake early-warning reporting times versus magnitudes of the $54(100 \%)$ correctly reported events.

\section{Early Warning Time of the Earthquake of Sep. 20, 1999 (Mw7.6)}

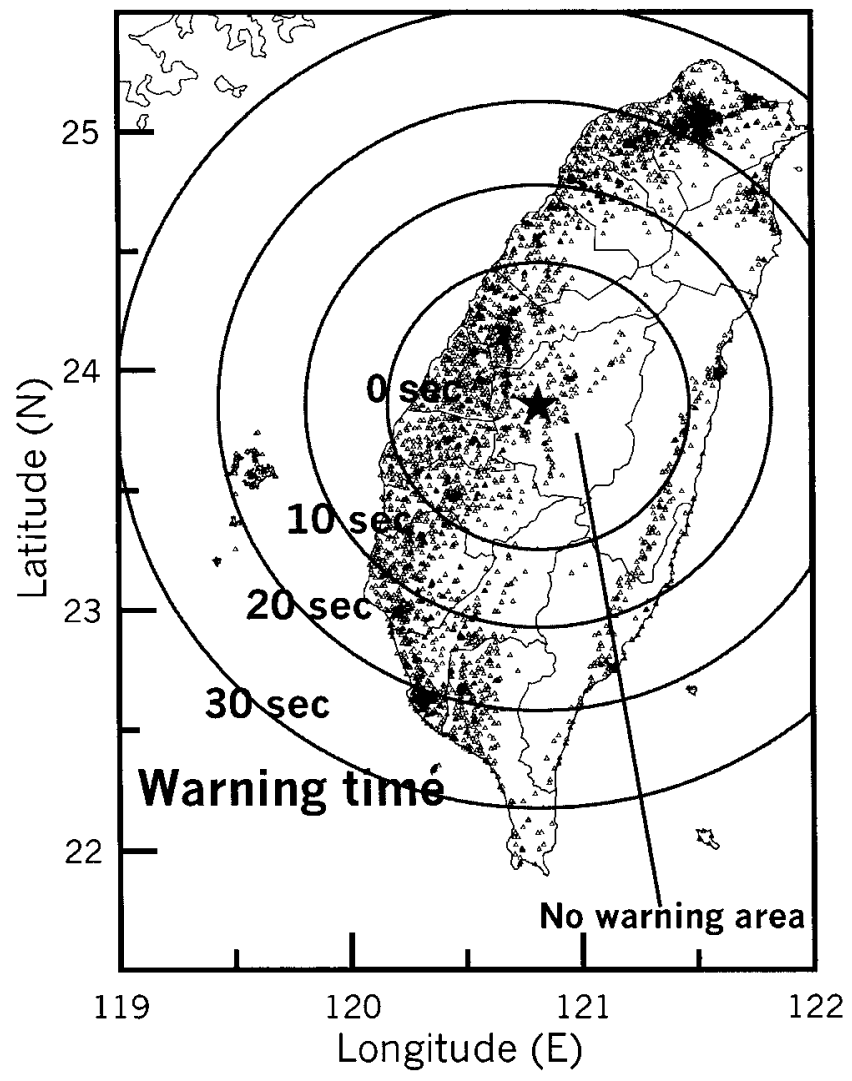

Figure 10. Expected VSN early-warning times (indicated by circles) in Taiwan with respect to the occurrence of an event similar to the Chi-Chi earthquake of 20 September 1999. Triangles give the locations of elementary schools, which can be regarded as the population density of Taiwan. 
assume that the off line manual measurements give correct values, our VSN results give an average error of $4.2 \pm 7.3$ $\mathrm{km}$ in epicenter, $4.5 \pm 5.5 \mathrm{~km}$ in focal depth, and an uncertainty of \pm 0.24 units in local magnitude. These values are small compared with the rupture lengths of the very large earthquakes at which RRS and EWS operations are targeted. In fact, for very large events such as the 1999 Chi-Chi earthquake, the rupture was $100 \mathrm{~km}$ long and the entire rupture process took nearly $40 \mathrm{sec}$, during which dislocations of a number of large asperities took place (Shin and Teng, 2001). The rupture of each large asperity will give rise to a set of destructive shear waves propagating outward. The rupture of the principal asperity will produce the most destructive shear waves from which the ultimate earthquake magnitude of a great event should be measured. Thus, for these large events, a 10-sec recording length will cover only the early portion of the rupture process in which the rupture of the principal asperity may or may not have taken place. Clearly, if we lengthen the recording time $T d$ to cover the entire (e.g., $40-\mathrm{sec}$ ) rupture process, there would have been no early warning to speak of.

In future developments, we need to implement the recursive moment-tensor-inversion algorithm for $M_{\mathrm{W}}$ determination of very large earthquakes. In addition, the $M_{\mathrm{W}}$ computation will need to be continually updated (say, every second) as the rupture is progressing in order to capture the principal energy release. Moreover, the Chi-Chi earthquake sequence (composed of many magnitude $>6.5$ events and one 7.6 event) allowed us to derive the strong-motion-attenuation curve and site-response data for Taiwan, based on recordings from a 650-station free-field, strong-motion network (Wu et al., 2001). These attenuation and site-response data will be incorporated into the RRS and EWS report to produce a refined map of the predicted strong shaking. These tasks are being pursued in Taiwan CWB and findings will be reported in due course.

As mentioned in the Introduction, the idea of an earlywarning system presented here takes advantage of the fast data-transmission speed (much faster than the seismic-wave speed) and fast data-processing speed. However, such a system has a "blind spot": it gives no warning to target areas closer than $50 \mathrm{~km}$ from the source. To provide early warning to areas close to the source, one would have to shrink the diameter of a VSN to one with impractical station density (a few-km station spacing) or to shrink the VSN to one with a single station. The UrEDAS (Nakamura, 1988, 1989) and the concept described by Grecksch and Kumpel (1997) explore such a single-station approach, which may provide some needed information sometime, but so far the singlestation approach yields excessive false alarms and large magnitude uncertainty. In Taiwan, we are studying a possible linear strong-motion array attached to the circum-island railroad system. Coupling the output of this linear-array system with that from the VSN system may eventually provide a more complete early-warning capability.

\section{Acknowledgments}

We wish to thank Chen-Chun Chen for his kind help for data preparation of this study. The authors also wish to thank William H. K. Lee, T. C. Shin, Yi-Ben Tsai, Jennifer Haye, and Chin-Chang Hung for reviewing this article and providing many thought-provoking comments. We thank David Oppenheimer and an anonymous reviewer for critical reviews and suggestions that helped clarify the presentation. One of us (Y.M.W.) was supported by the CWB and the National Science Council of the Republic of China under Grant Nos. NSC89-2625-Z-052-023, NSC90-2625-Z-052009, and MTOC-CWB-90-E-05. The other author (T.-1.T.) was supported by contract MTOC-CWB-90-E-05, NSC-90-2811-M-001-052 and NSF grants EAR-00010616, and EAR-0124926.

\section{References}

Espinosa-Aranda, J., A. Jiménez, G. Ibarrola, F. Alcantar, A. Aguilar, M. Inostroza, and S. Maldonado (1995). Mexico City seismic alert system, Seism. Res. Lett. 66, 42-53.

Grecksch, G., and H. J. Kumpel (1997). Statistical analysis of strongmotion accelerogram and its application to earthquake early-warning systems, Geophys. J. Int. 129, 113-123.

Hsu, M. T. (2001). Seismological observation and service in Taiwan (up to 1970), in Handbook of Earthquake and Engineering Seismology, W. H. K. Lee, H. Kanamori, and P. C. Jennings (Editors), New York, Academic, in press.

Kanamori, H., E. Hauksson, and T. Heaton (1997). Real-time seismology and earthquake hazard mitigation, Nature 390, 461-464.

Kanamori, H., P. Maechling, and E. Hauksson (1999). Continuous monitoring of ground-motion parameters, Bull. Seism. Soc. Am. 89, 311316.

Nakamura, Y. (1988). On the urgent earthquake detection and alarm system (UrEDAS), Proceedings of the 9th World Conference on Earthquake Engineering, Tokyo-Kyoto, Japan.

Nakamura, Y. (1989). Earthquake alarm system for Japan railways, Jpn Railway Eng. 109, $1-7$.

Qu, J., and T. L. Teng (1994). Recursive stochastic deconvolution in the estimation of earthquake source parameters: synthetic waveforms, Phys. Earth Planet. Interiors 86, 301-327.

Shin, T. C., and T. L. Teng (2001). An overview of the 1999 Chi-Chi, Taiwan, earthquake, Bull. Seism. Soc. Am. 91, 895-913.

Teng, T. L., Y. M. Wu, T. C. Shin, Y. B. Tsai, and W. H. K. Lee (1997), One minute after: strong-motion map, effective epicenter, and effective magnitude, Bull. Seism. Soc. Am. 87, 1209-1219.

Tsai, Y. B., and Y. M. Wu (1997). Quick determination of magnitude and intensity for seismic early warning, 29th IASPEI Meeting, Thessaloniki, Greece.

United States National Research Council (1991). Real-time earthquake monitoring: report from the Committee on Seismology, National Academy Press, Washington, DC, 52 pp.

United States Geological Survey (1998). A plan for implementing a realtime seismic hazard warning system-a report to Congress required by public law 105-47. March 27, 1998.

Wu, Y. M., C. C. Chen, T. C. Shin, Y. B. Tsai, W. H. K. Lee, and T. L. Teng (1997). Taiwan Rapid Earthquake Information Release System, Seism. Res. Lett. 68, 931-943.

Wu, Y. M., T. C. Shin, and Y. B. Tsai (1998a). Quick and reliable determination of magnitude for seismic early warning, Bull. Seism. Soc. Am. 88, 1254-1259.

Wu, Y. M., C. C. Chen, J. K. Chung, and T. C. Shin (1998b). An automatic phase picker of the real-time acceleration seismic network (in Chinese), Meteorol. Bull. 42, 103-117.

Wu, Y. M., J. K. Chung, T. C. Shin, N. C. Hsiao, Y. B. Tsai, W. H. K. Lee, and T. L. Teng (1999). Development of an integrated seismic 
early warning system in Taiwan-case for the Hualien area earthquakes, TAO 10, 719-736.

Wu, Y. M. (1999a). Development of real-time earthquake reporting and warning systems-Taiwan experience (in Chinese), Ph.D. Thesis, Institute of Geophysics, National Central University, 152 pp.

Wu, Y. M. (1999b). Development of event location software in an earthquake rapid reporting system, Central Weather Bureau Research and Development Report CW88-1A-08, 58 pp.

Wu, Y. M., W. H. K. Lee, C. C. Chen, T. C. Shin, T. L. Teng, and Y. B. Tsai (2000). Performance of the Taiwan Rapid Earthquake Information Release System (RTD) during the 1999 Chi-Chi (Taiwan) earthquake, Seism. Res. Lett. 71, 338-343.

Wu, Y. M., T. C. Shin, and C. H. Chang (2001). Near real-time mapping of peak ground acceleration and peak ground velocity following a strong earthquake, Bull. Seism. Soc. Am. 91, 1218-1228.

Central Weather Bureau

Taipei, Taiwan ROC

(Y.-M.W.)

Southern California Earthquake Center

University of Southern California

Los Angeles, California

(T.-1.T.)

Manuscript received 27 July 2001. 